\title{
Scaling test of quenched Wilson twisted mass QCD at maximal twist
}

DESY 05-189

\author{
K. Jansen, M. Papinutto*, A. Shindler, I. Wetzorke \\ John von Neumann-Institut für Computing NIC, Platanenallee 6, 15738 Zeuthen, Germany \\ E-mail: karl.jansen@desy.de, mauro.papinutto@desy.de, \\ andrea.shindlerddesy.de, ines.wetzorkeldesy.de \\ C. Urbach \\ John von Neumann-Institut für Computing NIC, Platanenallee 6, 15738 Zeuthen, Germany \& \\ Institut für Theoretische Physik, Freie Universität Berlin, Arnimallee 14, 14195 Berlin, Germany \\ E-mail: carsten.urbach@desy.de
}

\begin{abstract}
We present the results of an extended scaling test of quenched Wilson twisted mass QCD. We fix the twist angle by using two definitions of the critical mass, the first obtained by requiring the vanishing of the pseudoscalar meson mass $m_{\mathrm{PS}}$ for standard Wilson fermions and the second by requiring restoration of parity at non-zero value of the twisted mass $\mu$ and subsequently extrapolating to $\mu \rightarrow 0$. Depending on the choice of the critical mass we simulate at values of $\beta \in[5.7,6.45]$, for a range of pseudoscalar meson masses $250 \mathrm{MeV} \lesssim m_{\mathrm{PS}} \lesssim 1 \mathrm{GeV}$ and we perform the continuum limit for the pseudoscalar meson decay constant $f_{\mathrm{PS}}$ and various hadron masses (vector meson $m_{V}$, baryon octet $m_{\mathrm{oct}}$ and baryon decuplet $m_{\mathrm{dec}}$ ) at fixed value of $r_{0} m_{\mathrm{PS}}$. For both definitions of the critical mass, lattice artifacts are consistent with $\mathrm{O}(a)$ improvement. However, with the second definition, large $\mathrm{O}\left(a^{2}\right)$ discretization errors present at small quark mass with the first definition are strongly suppressed. The results in the continuum limit are in very good agreement with those from the Alpha and CP-PACS Collaborations.
\end{abstract}

XXIIIrd International Symposium on Lattice Field Theory

25-30 July 2005

Trinity College, Dublin, Ireland

\footnotetext{
${ }^{*}$ Speaker.
} 


\section{Introduction}

Twisted mass QCD (tmQCD), whose action reads

$$
S[U, \psi, \bar{\psi}]=a^{4} \sum_{x} \bar{\psi}(x)\left(D_{W}+m_{0}+i \mu \gamma_{5} \tau_{3}\right) \psi(x),
$$

has been proposed as an alternative to Wilson QCD because it is not affected by the problem of unphysical zero modes and can lead to simplifications of the operator mixing pattern [1]. At maximal twist (i.e. by setting $m_{0}$ to its critical value $m_{c}$ up to $\mathrm{O}(a)$, with $\mu$ now the bare quark mass) it has been shown [2] that parity even correlators (and thus energies and matrix elements) are automatically $\mathrm{O}(a)$ improved. Due to these properties, tmQCD is a very interesting candidate for dynamical simulations at small quark masses [3]. However, right at small quark masses $\mu \lesssim a^{1}$, large lattice artifacts have been observed when using a definition of $m_{c}$ obtained by requiring the vanishing of $m_{\mathrm{PS}}^{2}$ with standard Wilson fermions (in the following we will call it $m_{c}^{\text {pion }}$ ) [4]. This kind of lattice artifacts are obviously affecting also dynamical simulations. An analysis based on Wilson Chiral Perturbation Theory (W $\chi$ PT) [5] suggested a definition of $m_{c}$ suitable to reach quark masses $\mu \sim a^{2}$. This definition is obtained by requiring the vanishing of the PCAC quark mass

$$
m_{\mathrm{PCAC}}=\lim _{x_{0} \rightarrow \infty} \frac{\sum_{\mathbf{x}}\left\langle\partial_{0} A_{0}^{a}(x) P^{a}(0)\right\rangle}{2 \sum_{\mathbf{x}}\left\langle P^{a}(x) P^{a}(0)\right\rangle}
$$

where $P^{a}=\bar{\psi} \gamma_{5} \frac{\tau^{a}}{2} \psi, A_{\mu}^{a}=\bar{\psi} \gamma_{\mu} \gamma_{5} \frac{\tau^{a}}{2} \psi$

An analysis à la Symanzik beyond $\mathrm{O}(a)$ shows the presence of $\mathrm{O}\left(a^{2}\right)$ cutoff effects enhanced at small pion mass, the most dangerous of which are of order $\left(a / m_{\mathrm{PS}}^{2}\right)^{2 k} k \geq 1$ which signal the presence of possibly large lattice artifacts in the $m_{\mathrm{PS}}^{2} \lesssim a$ regime [6] (see also [7]). The result of this analysis is that there are two ways of reducing these large lattice artifacts: fixing the $\mathrm{O}(a)$ ambiguity in $m_{c}$ as proposed in Refs. [5] (we will call this definition $m_{c}^{\mathrm{PCAC}}$ ) or add the clover term with non-perturbatively determined $c_{S W}$ coefficient (and the corresponding value of $m_{c}$ ). We investigate here the first proposal (see Refs. [8] for the second possibility).

\section{Determination of $m_{c}^{\mathrm{PCAC}}$}

$m_{\mathrm{PCAC}}$ depends, in the neighborhood of a given estimate of $m_{c}$ (e.g. $\left.m_{c}^{\text {pion }}\right)$, smoothly on both $m_{0}$ and $\mu$. Moreover, in the quenched approximation, a multiple mass solver can be used to compute the fermion propagator for different $\mu$ values at a given value of $m_{0}$. In view of these two facts, the procedure we adopted to determine $m_{c}^{\mathrm{PCAC}}$ is the following [9]:

1. choose $n$ values of $\mu$ (with $\mu>a^{2}$ ) and $n^{\prime}$ values of $m_{0}$ (in the vicinity of $m_{c}^{\text {pion }}$ ) which cover the range where $m_{\mathrm{PCAC}}(\mu)$ is close to zero (in the present case $n=9$ and $n^{\prime}=4$ ).

2. find the values of $m_{c}(\mu)$ at which $m_{\mathrm{PCAC}}(\mu)$ is zero (see Fig. 1.a)

3. extrapolate $m_{c}(\mu)$ to $\mu=0$ (see Fig. 1.a) ${ }^{2}$.

In Ref. [6] one can find a theoretical analysis that justifies this procedure and shows that the value of $m_{c}^{\mathrm{PCAC}}$ in which we are interested can be obtained by a linear extrapolation from the region $\mu>a^{2}$ down to $\mu=0 . m_{c}^{\mathrm{PCAC}}$ has been determined for $\beta \in\{5.7,5.85,6.0,6.2\}$ using statistics of $\underline{\mathrm{O}(100)-\mathrm{O}(200) \text { gauge configurations. }}$

\footnotetext{
${ }^{1}$ powers of $\Lambda_{\mathrm{QCD}}$ required to match physical dimensions are in the following understood
}

${ }^{2}$ in Ref. [10] for each simulated value of $\mu$ the corresponding value of $m_{c}(\mu)$ were used. 

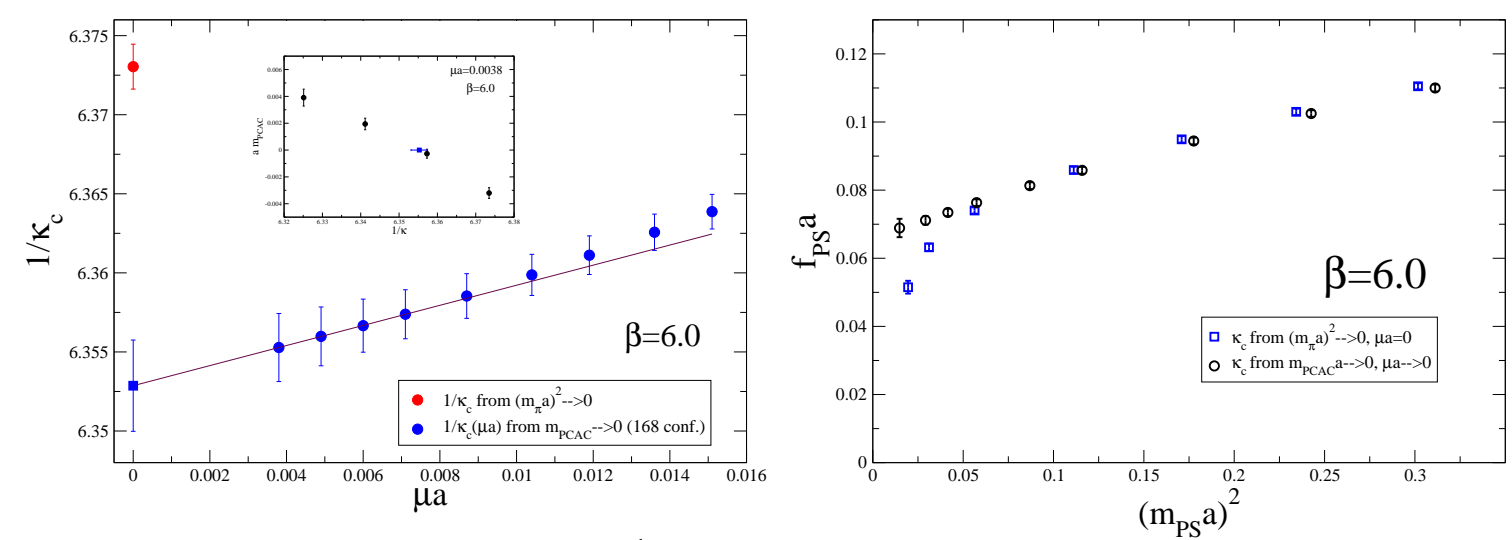

Figure 1: a. Determination of $m_{c}(\mu)\left(\kappa_{c}^{-1}=2 a m_{c}+8\right)$ for given values of $\mu$ and $\beta$ and extrapolation of $m_{c}(\mu)$ to $\mu=0$. b. $f_{\mathrm{PS}}$ as function of $m_{\mathrm{PS}}^{2}$ at $\beta=6.0$ for the two definitions of $m_{c}\left(m_{c}^{\text {pion }}\right.$ and $\left.m_{c}^{\mathrm{PCAC}}\right)$.

\section{Chiral behaviour at fixed $a$}

The effectiveness of $m_{c}^{\mathrm{PCAC}}$ in reducing the large lattice artifacts observed at small quark mass is evident when considering the chiral behaviour of two simple observables: $f_{\mathrm{PS}}$ and $m_{\mathrm{PS}} . f_{\mathrm{PS}}$ can be extracted, without need of renormalization constants, by using the exact lattice PCVC relation $\left\langle\partial_{\mu}^{*} \tilde{V}_{\mu}^{a}(x) O(0)\right\rangle=-2 \mu \varepsilon^{3 a b}\left\langle P^{b}(x) O(0)\right\rangle$ (where $\partial_{\mu}^{*}$ is the lattice backward derivative and $\tilde{V}_{\mu}^{a}$ the point-splitted vector current). It follows that, at maximal twist,

$$
f_{\mathrm{PS}}=\frac{2 \mu}{m_{\mathrm{PS}}^{2}}\left|\left\langle 0\left|P^{a}\right| P S\right\rangle\right| .
$$

In Fig. 1.b one can compare the chiral behaviour of $f_{\mathrm{PS}}$ obtained with either $m_{c}^{\text {pion }}$ or $m_{c}^{\mathrm{PCAC}}$. One sees immediately that $m_{c}^{\mathrm{PCAC}}$ reduces the large lattice artifacts present at small $\mu$ when using $m_{c}^{\text {pion }}$ ( $f_{\mathrm{PS}}$ is predicted to be linear in $m_{\mathrm{PS}}^{2}$ at one loop in quenched $\chi \mathrm{PT}$ ).

Using the integrated PCVC relation, it is also possible to prove [6] that, by using $m_{c}^{\mathrm{PCAC}}$ and in the region $\mu>a^{2}, m_{P S}^{2}$ is linear with $\mu$ up to small $a^{4}$ cut-off effects ${ }^{3}$ (i.e. $\mathrm{O}\left(a^{2}\right)$ lattice artifacts are proportional to $\mu$ ). This is qualitatively confirmed by our data as shown in Fig. 2.a.

\section{Scaling behaviour}

We present now results concerning the scaling behaviour of $f_{\mathrm{PS}} r_{0}, m_{V} r_{0}, m_{\mathrm{oct}} r_{0}$ and $m_{\mathrm{dec}} r_{0}$ at fixed value of $m_{\mathrm{PS}} r_{0}$ (where $r_{0}$ is the Sommer scale). More details concerning meson quantities can be found in Ref. [11]. $m_{V}$ has been extracted by using either the spatial component of the axial vector or the temporal component of the tensor as interpolating operators (in the following we will quote only the latter, which systematically present lower statistical errors). $m_{\mathrm{oct}}$ and $m_{\mathrm{dec}}$ have been extracted by using respectively $\varepsilon^{A B C}\left(\left(d^{A}\right)^{T} C \gamma_{5} u^{B}\right) u_{\alpha}^{C}$ and $\varepsilon^{A B C}\left(\left(u^{A}\right)^{T} C \gamma_{k} u^{B}\right) u_{\alpha}^{C}$ as interpolating operators. The parameters of the simulations can be found in Tab. 1. We have simulated quark masses $\mu$ corresponding to $235 \mathrm{MeV} \leq m_{P S} \leq 1.0 \mathrm{GeV}$ (where the scale $r_{0}$, as will be explained below, has been fixed through the $\rho$ mass). The scaling behaviour of $f_{\mathrm{PS}} r_{0}$, as shown in Fig. 2.b, is clearly linear in $\left(a / r_{0}\right)^{2}$. However, $m_{c}^{\text {pion }}$ gives large $\mathrm{O}\left(a^{2}\right)$ effects at small masses, effects that are

\footnotetext{
${ }^{3}$ chiral logs and other $\mathrm{O}\left(m_{\mathrm{PS}}^{2}\right)$ contributions are here assumed to be negligible
} 

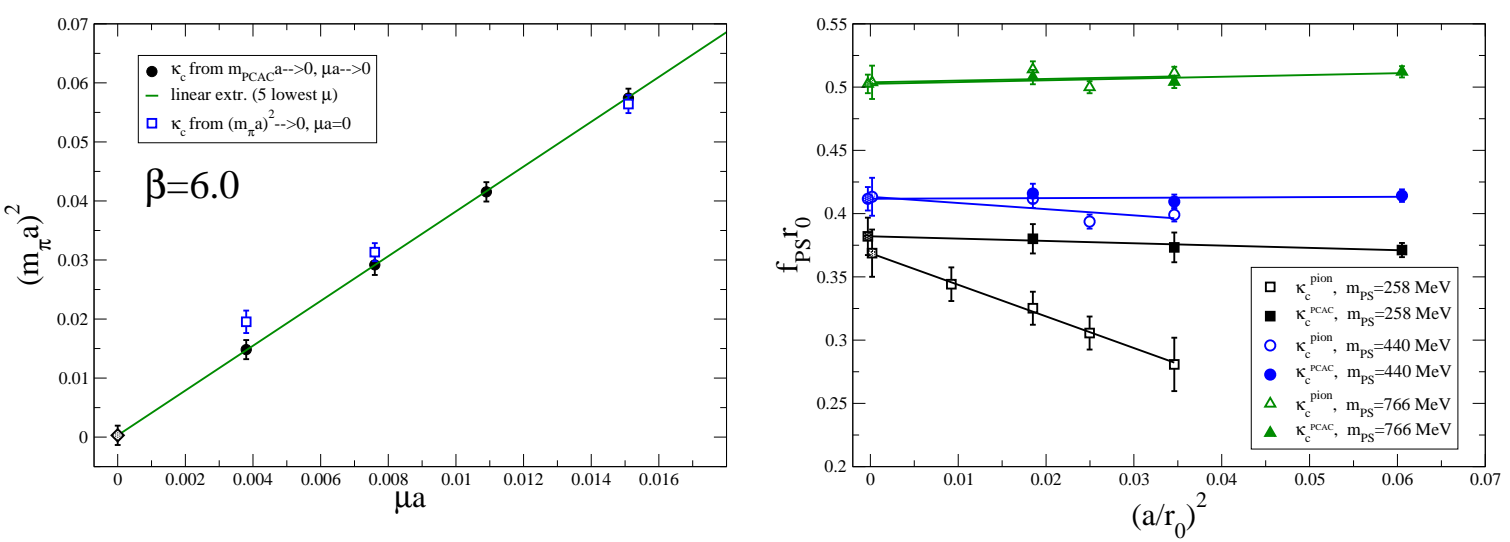

Figure 2: a. $m_{\mathrm{PS}}$ as function of $\mu$ at $\beta=6.0$ for both definitions $m_{c}^{\text {pion }}$ and $m_{c}^{\mathrm{PCAC}} . \mathrm{b}$. Scaling behaviour of $f_{\mathrm{PS}} r_{0}$ for 3 fixed values of $m_{\mathrm{PS}} r_{0}$. For each definition of $m_{c}$ an indipendent fit has been performed.

drastically reduced by the use of $m_{c}^{\mathrm{PCAC}}$. This obviously influences the scaling region which starts at $\beta=6.0$ for $m_{c}^{\text {pion }}$ and at $\beta=5.85$ for $m_{c}^{\mathrm{PCAC}}$. We perform indipendent continuum extrapolations for the two choices of $m_{c}$ and the results are in good agreement. In the case of $m_{c}^{\text {pion }}$, due to the highest slope for the lowest quark mass, we needed an additional point at $\beta=6.45$ in order to control the extrapolation. $m_{V} r_{0}, m_{\mathrm{oct}} r_{0}$ and $m_{\mathrm{dec}} r_{0}$ have been thus computed by using only $m_{c}^{\mathrm{PCAC}}$.

Since we have simulated down to $m_{\mathrm{PS}}$ of $235 \mathrm{MeV}$, finite size effects (FSE) can be quite relevant. In order to check for FSE we performed two additional simulations at $\beta=5.85$ on volumes of $12^{3} \times 32$ and $14^{3} \times 32$ in order to extend the results of Ref. [13] at smaller masses. For meson quantities ( $m_{\mathrm{PS}}, f_{\mathrm{PS}}$ and $\left.m_{V}\right)$ FSE are negligible for all the quark masses starting from the third smallest one; on the two smallest masses they are in practice below the statistical accuracy of our data. For baryon masses, instead, FSE are very large for the two smallest masses and still relevant on the next three. Since the sensitivity required to study FSE on the smallest two masses is computationally very expensive, we chose here to correct only the data from the third value of $\mu$ on (corresponding to $m_{\mathrm{PS}}=375 \mathrm{MeV}$ ). The results for the scaling behaviour of $m_{\mathrm{oct}} r_{0}$ are shown in Fig. 3.a from which the lattice artifacts appear to be $\mathrm{O}\left(a^{2}\right)$ down to $\beta=5.85$ and of relatively small size.
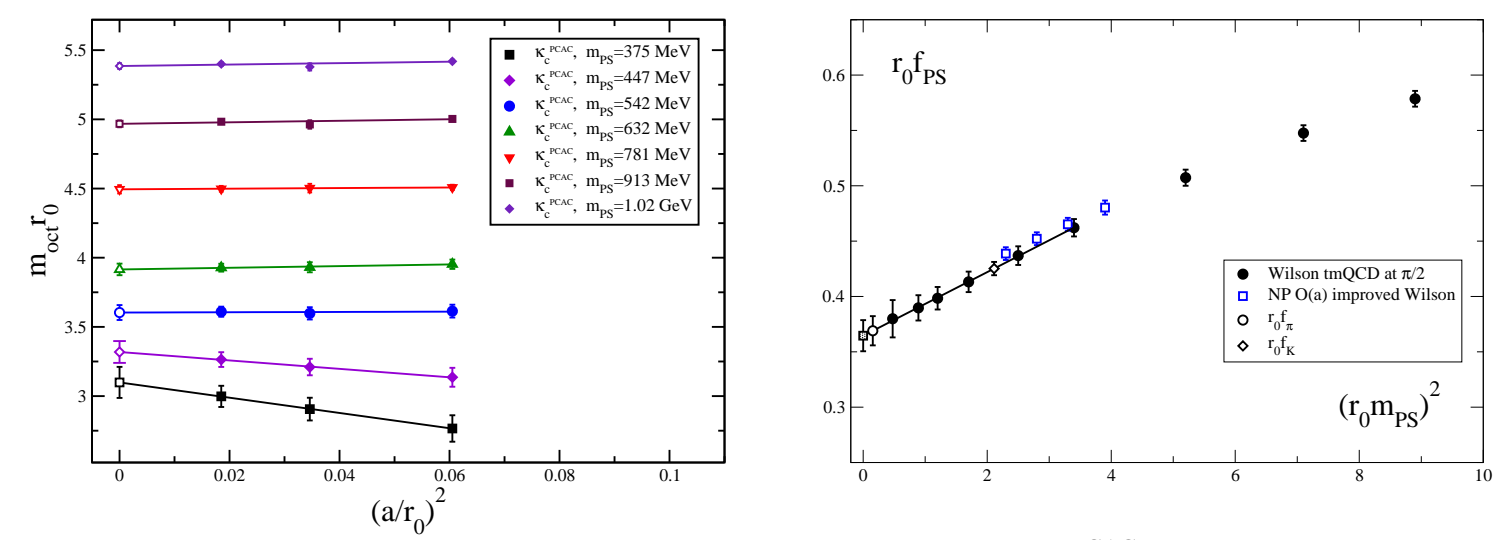

Figure 3: a. Scaling behaviour of $m_{\mathrm{oct}} r_{0}$ for 7 fixed values of $m_{\mathrm{PS}} r_{0}$ (only $m_{c}^{\mathrm{PCAC}}$ used). b. Continuum limit of $f_{\mathrm{PS}} r_{0}$ as function of $\left(m_{\mathrm{PS}} r_{0}\right)^{2}$ (only $m_{c}^{\mathrm{PCAC}}$ used). The empty squares are results taken from [12] 


\begin{tabular}{|c||c|c|c|c|c|c|}
\hline \hline$\beta$ & 5.70 & 5.85 & 6.00 & 6.10 & 6.20 & 6.45 \\
\hline$a(\mathrm{fm})$ & 0.171 & 0.123 & 0.093 & 0.079 & 0.068 & 0.048 \\
$L / a$ & 12 & 16 & 16 & 20 & 24 & 32 \\
$T / a$ & 32 & 32 & 32 & 40 & 48 & 64 \\
\hline$N_{\text {conf }}\left(m_{c}^{\text {pion }}\right)$ & 600 & 378 & 387 & 300 & 260 & 182 \\
\hline$N_{\text {conf }}\left(m_{c}^{\text {PCAC }}\right)$ & 600 & 500 & 400 & & 300 & \\
\hline \hline
\end{tabular}

Table 1: Parameters of the simulations

\section{Continuum limit}

Results for the continuum limit of $f_{\mathrm{PS}} r_{0}, m_{V} r_{0}, m_{\mathrm{oct}} r_{0}$ and $m_{\mathrm{dec}} r_{0}$ are presented in Fig. 3.b and 4.a. Our determinations of $f_{\mathrm{PS}}$ and $m_{V}$ are in good agreement with those from the ALPHA Coll. [12] with non-perturbatively $\mathrm{O}(a)$ improved Wilson fermions (the comparison for $f_{\mathrm{PS}}$ is shown in Fig. 3.b). For the chiral extrapolation we used the form $f_{P S}, m_{V} \sim A+B m_{P S}^{2}$ and $m_{\text {oct }}$, $m_{\mathrm{dec}} \sim A+B m_{P S}+C m_{P S}^{2}$. In order to compare with the results of the CP-PACS Coll. [14], we fixed the scale $r_{0}$ through the $\rho$ mass $m_{\rho}$, obtaining $r_{0}=0.576 \mathrm{fm}^{4}$. As a prediction we get $f_{\pi}, m_{N}, m_{\Delta}$ and (working in the $S U(3)$ symmetric limit) $f_{K}$ and $m_{K^{*}}$. The results can be found in Fig. 4.b and Tab. 2 together with those of Ref. [14] and turn out to be in very good agreement. Notice however that in the present work quantities are $\mathrm{O}(a)$ improved and pseudoscalar masses significantly smaller than those in Ref. [14] (where standard Wilson fermions were used) have been simulated.

\section{Conclusions and Outlook}

In the present study we have extrapolated to the continuum (taking into account possible FSE) meson quantities $\left(f_{P S}\right.$ and $\left.m_{V}\right)$ and baryon masses $\left(m_{\mathrm{oct}}\right.$ and $\left.m_{\mathrm{dec}}\right)$ down to pseudoscalar messon masses of $235 \mathrm{MeV}$ and $375 \mathrm{MeV}$ respectively. We have presented a strong evidence that lattice artifacts are $\mathrm{O}\left(a^{2}\right)$ for both definitions of $m_{c}\left(m_{c}^{\text {pion }}\right.$ and $\left.m_{c}^{\mathrm{PCAC}}\right)$ and moreover that the use of $m_{c}^{\mathrm{PCAC}}$ drastically reduces the chirally enhanced $\mathrm{O}\left(a^{2}\right)$ lattice artifacts present at small quark masses when
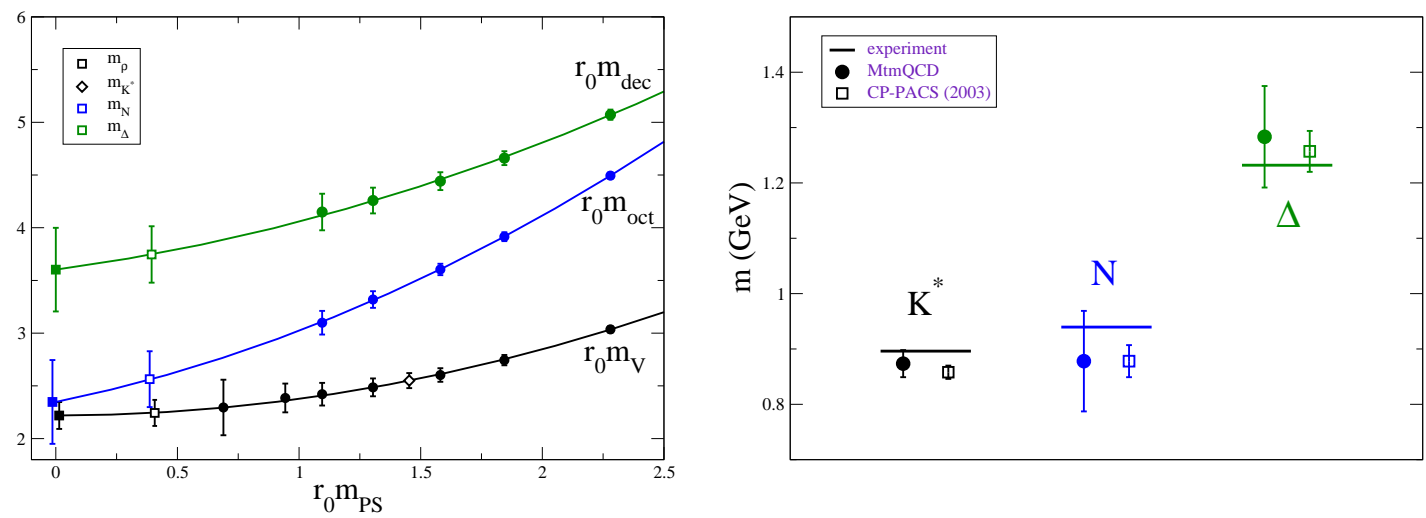

Figure 4: a. Continuum limit of $m_{V} r_{0}, m_{\mathrm{oct}} r_{0}, m_{\mathrm{dec}} r_{0}$ as function of $m_{\mathrm{PS}} r_{0}$ (only $m_{c}^{\mathrm{PCAC}}$ used). b. Comparison of our results for $m_{K^{*}}, m_{N}, m_{\Delta}$ with those of Ref. [14].

\footnotetext{
${ }^{4}$ had we fixed the scale through $f_{K}$ we would have obtained $r_{0}=0.508 \mathrm{fm}$
} 


\begin{tabular}{|c||c|c|c|}
\hline \hline & $f_{\pi}(\mathrm{MeV})$ & $f_{K}(\mathrm{MeV})$ & $f_{K} / f_{\pi}$ \\
\hline exp. & 132 & 160 & 1.22 \\
tmQCD & $126(5)$ & $146(3)$ & $1.15(5)$ \\
CP-PACS & $120(6)$ & $139(5)$ & $1.16(3)$ \\
\hline \hline
\end{tabular}

Table 2: Pseudoscalar meson decay constants from the present work and from Ref. [14] (in Ref. [14], tadpole-improved perturbation theory has been used to renormalize the axial current).

using $m_{c}^{\text {pion }}$. Our results for the continuum extrapolated quantities are in good agreement with those from the ALPHA [12] and CP-PACS [14] Collaborations. This is very encouraging in view of future dynamical simulations [3]. There are however other aspects of tmQCD that it is worth to investigate, for instance the problem of isospin breaking (see Ref. [15] for an exploratory study in the quenched approximation). This problem is practically very important for phenomenological applications of tmQCD and also strictly related to the phase structure of tmQCD in the neighborhood of the critical point $[5,16]$ and thus directly relevant for dynamical symulations.

\section{References}

[1] R. Frezzotti, P. A. Grassi, S. Sint, and P. Weisz, JHEP 08 (2001) 058;

R. Frezzotti, Nucl. Phys. Proc. Suppl. 119 (2003) 140.

[2] R. Frezzotti and G. C. Rossi, JHEP 08 (2004) 007;

R. Frezzotti, Nucl. Phys. Proc. Suppl. 140 (2005) 134.

[3] A. Shindler, these Proceedings;

F. Farchioni, N. Ukita, U. Wenger, I. Wetzorke, these Proceedings.

[4] W. Bietenholz et al. ( $\chi_{\mathrm{L}} \mathrm{F}$ Coll.), JHEP 12 (2004) 044.

[5] S. Aoki and O. Bar, Phys. Rev. D70 (2004) 116011;

S. R. Sharpe and J. M. S. Wu, Phys. Rev. D71 (2005) 074501.

[6] R. Frezzotti, G. Martinelli, M. Papinutto, and G. C. Rossi, [hep-lat/0503034];

G. C. Rossi, these Proceedings.

[7] S. R. Sharpe, [hep-lat/0509009].

[8] M. Della Morte, R. Frezzotti and J. Heitger, Nucl. Phys. Proc. Suppl. 106 (2002) 260;

V. Lubicz, these Proceedings.

[9] K. Jansen, M. Papinutto, A. Shindler, C. Urbach, I. Wetzorke ( $\chi_{\mathrm{L}}$ F Coll.), Phys. Lett. B619 (2005) 184.

[10] A. M. Abdel-Rehim, R. Lewis, and R. M. Woloshyn, Phys. Rev. D71 (2005) 094505.

[11] K. Jansen, A. Shindler, C. Urbach, I. Wetzorke ( $\chi_{\mathrm{L}} \mathrm{F}$ Coll.), Phys. Lett. $\mathbf{B 5 8 6}$ (2004) 432;

K. Jansen, M. Papinutto, A. Shindler, C. Urbach, I. Wetzorke ( $\chi_{\mathrm{L}} \mathrm{F}$ Coll.), [hep-lat $\left./ 0507010\right]$.

[12] J. Garden, J. Heitger, R. Sommer, H. Wittig (ALPHA Coll.), Nucl. Phys. B571 (2000) 237.

[13] M. Guagnelli et al. (ZeRo Coll.), Phys. Lett. B597 (2004) 216;

I. Wetzorke, K. Jansen, F. Palombi, A. Shindler, Nucl. Phys. Proc. Suppl. 140 (2005) 393.

[14] S. Aoki et al. (CP-PACS Coll.), Phys. Rev. D67 (2003) 034503.

[15] K. Jansen et al. ( $\chi_{\mathrm{L}} \mathrm{F}$ Coll.), Phys. Lett. $\mathbf{B 6 2 4}$ (2005) 334, [hep-lat 0507032 ];

J. Pickavance, these Proceedings.

[16] L. Scorzato, Eur. Phys. J. C37 (2004) 445. 